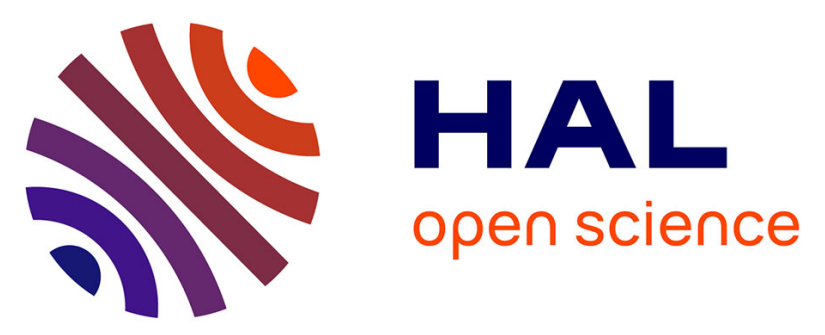

\title{
New density profile reconstruction methods in X-mode reflectometry
}

\author{
Rennan Bianchetti Morales, S. Hacquin, Stéphane Heuraux, R. Sabot
}

\section{To cite this version:}

Rennan Bianchetti Morales, S. Hacquin, Stéphane Heuraux, R. Sabot. New density profile reconstruction methods in X-mode reflectometry. Review of Scientific Instruments, 2017, 88, pp.043503. 10.1063/1.4979513. hal-01580343

\section{HAL Id: hal-01580343 \\ https://hal.univ-lorraine.fr/hal-01580343}

Submitted on 6 May 2020

HAL is a multi-disciplinary open access archive for the deposit and dissemination of scientific research documents, whether they are published or not. The documents may come from teaching and research institutions in France or abroad, or from public or private research centers.
L'archive ouverte pluridisciplinaire HAL, est destinée au dépôt et à la diffusion de documents scientifiques de niveau recherche, publiés ou non, émanant des établissements d'enseignement et de recherche français ou étrangers, des laboratoires publics ou privés. 


\title{
New density profile reconstruction methods in X-mode reflectometry
}

\author{
R. B. Morales, ${ }^{1, \text { a) }}$ S. Hacquin, ${ }^{2,3}$ S. Heuraux,${ }^{1}$ and R. Sabot ${ }^{2}$ \\ ${ }^{1}$ IJL, University of Lorraine, UMR 7198 CNRS, 54506 Vandoeuvre, France \\ ${ }^{2}$ IRFM, CEA, Cadarache, 13108 Saint-Paul-lez-Durance, France \\ ${ }^{3}$ EUROfusion Programme Management Unit, Culham Science Centre, Culham, OX14 3DB, United Kingdom \\ ]
}

The reconstruction method published by Bottollier-Curtet and Ichtchenko in 1987 has been the standard method of density profile reconstruction for X-mode reflectometry ever since, with only minor revision. Envisaging improved accuracy and stability of the reconstruction method, functions more complex than the linear are evaluated here to describe the refractive index shape in each integration step. The stability and accuracy obtained when using parabolic and fixed or adaptative fractional power functions are compared to the previous method and tested against spurious events and phase noise. The developed relation from the plasma parameters to the best integration shapes allows for the optimization of the reconstruction for any profile shape. In addition, the density profiles can be reconstructed using less probing frequencies without accuracy loss, which speed up the reconstruction algorithm and enable real-time monitoring of faster density profile evolution.

[http://dx.doi.org/10.1063/1.4979513]

\section{INTRODUCTION}

Together with the evolution of the nuclear fusion experiments comes a demand for more accurate determination of the plasma properties, such as the density profile. Having a more accurate density profile enables more refined analysis of the plasma turbulence properties, which is relevant for confinement investigation and monitoring. In addition, the speed of the profile reconstruction process is also crucial for real-time monitoring of the plasma position for safety reasons. The X-mode microwave reflectometry is a well established non-invasive technique able to probe the plasma at the high temperature and density environment of current and next generation experiments. The development of several reflectometry systems is included in the ITER project. One relevant system to the topics developed here is the low field side X-mode reflectometer. Its first operation priority is to measure the density profile with the desired minimum spatial accuracy of $5 \mathrm{~mm} .{ }^{1}$

The theoretical maximum experimental accuracy of a reconstructed profile has already been shown. ${ }^{2,3}$ However, no systematic evaluation of the accuracy from the data analysis point exists to date. In order to evaluate and improve this accuracy, three aspects can be explored: first, the initialization of the profile reconstruction, currently performed as published in the Refs. 4 and 5; second, the recursive method used to compute the reflection positions of each probing frequency; and third, the identification and description of perturbations that introduce blind zones to the reflectometer. This situation can occur in the core plasma during the start up of heating systems, ${ }^{6}$ massive gas and pellet injections, ${ }^{7}$ for hollow profiles ${ }^{6}$ and MHD activity, ${ }^{8}$ and in edge turbulence, such as filaments or blobs. ${ }^{9}$ The latter being also an issue for the initialization of the reconstruction.
The initialization methods currently employed are subject to an error of up to $1 \mathrm{~cm}^{4}$ On the other hand, the description of blind areas has never been taken into account so far. Treating these two topics is beyond the scope of this paper. Dedicated full-wave simulations are being performed to seek improvement in these topics and dedicated papers are necessary.

This paper is devoted to improving the density profile reconstruction method using X-mode reflectometry data following the ideas presented in the Refs. 10-12. It is theoretically possible to increase the accuracy of the reconstructed profile simply by increasing the number of frequencies considered for the reconstruction. However, the practical frequency resolution is limited not only by the resolution of the equipment but also, more importantly, by the presence of many sources of noise. Furthermore, real-time monitoring applications benefit from faster reconstruction algorithms which have a direct relation to the number of frequency steps used in the reconstruction. Instead of increasing the number of probing frequencies, it is investigated here how to improve the position evaluation of each frequency step. Only synthetic data are used throughout this paper. This is the only way to know the true density profile, thus enabling the accuracy of the applied reconstruction method to be verified.

\section{OVERVIEW OF THE RECONSTRUCTION METHOD OF BOTTOLLIER-CURTET}

Two cutoff branches are available to probe with the Xmode polarization: the left-hand polarization in lower frequencies and right-hand polarization in higher frequencies. These cutoff frequency profiles are given by ${ }^{13}$

$$
f_{L, R}=\frac{\mp f_{c}+\sqrt{f_{c}^{2}+4 f_{p}^{2}}}{2}
$$


with

$$
f_{c}=\frac{e B}{2 \pi m_{e}}, \quad f_{p}=\frac{1}{2 \pi} \sqrt{\frac{n_{e} e^{2}}{m_{e} \epsilon_{0}}},
$$

where $f_{L}$ is the left-hand polarization cutoff frequency corresponding to the minus sign, $f_{R}$ is the right-hand polarization cutoff frequency corresponding to the plus sign, $f_{c}$ the electron cyclotron frequency, $f_{p}$ the plasma frequency, $e$ the electron charge, $m_{e}$ the electron mass, $B$ the magnetic field, $n_{e}$ the electron density, and $\epsilon_{0}$ the vacuum permittivity. Furthermore, the dispersion relation reads

$$
N=\sqrt{1-\frac{X(1-X)}{1-X-Y^{2}}}
$$

with

$$
X=\frac{f_{p}^{2}}{f^{2}}, \quad Y=\frac{f_{c}}{f},
$$

where $N$ is the refractive index and $f$ the injected frequency.

The reconstruction method of Bottollier-Curtet ${ }^{14}$ computes the spatial step from the phase shift associated with the frequency step. To find the phase shift, one compares the measured phase shift (including the total path of the probing waves) to the numerically calculated phase shift under the WKB approximation up to the last known position. A representation of each of these components is found in Fig. 1. The numerical phase shift from point $A$ to $B$ is computed integrating the profile of refractive index, as in Eq. (5), ${ }^{15}$

$$
\phi(B)-\phi(A)=\frac{2 \pi f}{c} \int_{A}^{B} N\left(f, f_{p}(x), f_{c e}(x)\right) d x,
$$

where $N$ is the refractive index, $f$ is the launched wave frequency, and $c$ is the speed of light in vacuum.

In the case of $\mathrm{X}$-mode reflectometry, the integral of the refractive index is too complex to be determined analytically. A method like the well established Abel inversion for the O-mode polarization cannot be developed in this case. The algorithm developed by Bottollier-Curtet assumed a linear refractive index profile between the known positions, and therefore a trapezoid integration is used across the entire profile. For the trapezoidal integration scheme, the area under the refractive

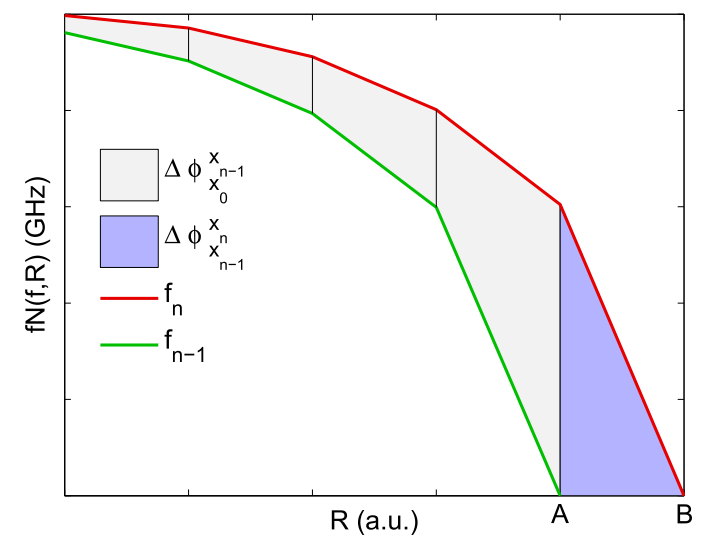

FIG. 1. Reconstruction scheme comparing the phase shift to the trapezoidal integration of the injected frequency times the refractive index as in Eq. (5), as a function of the plasma radius starting at the edge and going towards the core, based on Bottollier-Curtet's paper. ${ }^{14}$ index in the last spatial step can be calculated as the area of a right triangle. The next position, $x_{n}$, in terms of the previous position, $x_{n} 1$, and the refractive index $N_{n} 1$ at position $x_{n}$, reads, according to Eq. (5),

$$
\begin{aligned}
& \Delta \phi_{x_{n-1}}^{x_{n}}=W \frac{2 \pi f_{n}}{c} N\left(f_{n}, x_{n-1}\right) \times\left(x_{n}-x_{n-1}\right), \\
& \therefore x_{n}=x_{n-1}+\frac{c \Delta \phi_{x_{n-1}}^{x_{n}}}{W 2 \pi f_{n} N\left(f_{n}, x_{n-1}\right)}
\end{aligned}
$$

with a weight factor $W=1 / 2$ for the linear case and $\Delta \phi_{x_{n-1}}^{x_{n}}$ being the total phase shift of a frequency step $f_{n-1}$ to $f_{n}$. Explicitly from the evaluation of Fig. 1, $\Delta \phi_{x_{n-1}}^{x_{n}}$ can be written as

$$
\Delta \phi_{x_{n-1}}^{x_{n}}=\Delta \phi_{f_{n-1}}^{f_{n}}-\left[\phi_{x_{0}}^{x_{n-1}}\left(f_{n}\right)-\phi_{x_{0}}^{x_{n-1}}\left(f_{n-1}\right)\right],
$$

with $\nabla \phi_{f_{n-1}}^{f_{n}}$ being the experimental measurement and $\left[\phi_{x_{0}}^{x_{n-1}}\left(f_{n}\right)-\phi_{x_{0}}^{x_{n-1}}\left(f_{n-1}\right)\right]$ numerically computed.

Reconstructing the profile with the recursive Eq. (6) and the weight factor $W=1 / 2$ results in an unstable reconstruction method. A stabilization factor was introduced for the reconstruction to converge. The numerically computed phase shift of each frequency step was averaged with the previous step. Considering approximations in the phase expression, it was later proposed by Bottollier-Curtet ${ }^{16}$ that in the vicinity of the reflection point (where the refractive index goes to zero), the integral of the refractive index reduces to $W=2 / 3$, instead of the $1 / 2$ from the trapezoidal case. The same result was obtained later when the trapezoidal integration was performed over the dielectric permittivity instead of the refractive index, as proposed by Shelukhin. ${ }^{17}$ Following these results, the methodology remains to integrate all the previously determined positions with the trapezoidal scheme and only the last position with the integration weight factor of $2 / 3$. This is valid for every frequency step computed.

\section{NEW APPROACHES ON THE RECONSTRUCTION METHOD}

When the probing wave is propagating with a frequency well above the local cutoff frequency, the refractive index is almost constant at the value of one. In this case, the trapezoidal scheme is accurate. On the other hand, near the reflection position, the refractive index changes abruptly to the value of zero, and it is not well described by a linear function. Envisaging a reconstruction which is more accurate, stable, and less sensitive to noise and turbulence, the usage of functions more complex than linear in the last integration step of the refractive index is investigated. The first step to improve the linear shape was to implement parabolic shapes. The parabolas are found from the local plasma properties, meaning that the used parabola is different at each frequency step. Then a square root profile is assumed for all frequency steps, since it has the same behaviour of the refractive index (high gradient close to zero). Lastly, functions of the type $x^{\alpha}$ are assumed, with $\alpha$ between zero and one, depending on the local plasma parameters. The Secs. III A-III C describe how to implement these integration shapes, followed by a comparison of the accuracy and reconstruction stability obtained on different profile shapes. 


\section{A. Parabolic integration shapes}

Three parameters are necessary to determine a parabolic profile for each integration step. Furthermore, the position of the cutoff is not yet known, and in fact, it is the parameter to be computed $\left(x_{n}\right)$. Thus, in total, four boundary conditions are assumed. They are based on the previous radial positions already reconstructed $\left(x_{i}\right)$. They are as follows: (1) the refractive index area under the last integration step $\left(\int_{x_{n-1}}^{x_{n}} N d x\right)$, which is determined from the phase shift measurement; (2) the refractive index is zero at the reflection position; (3) the point (position, refractive index) from the last radial position already reconstructed; and (4) the point (position, refractive index) from one radial position before the last already reconstructed position. An additional boundary condition can be the continuity of the refractive index slope at the position prior to the reflection point. This condition was also assumed in the development process, leading to an additional parabolic method where this condition replaced the condition 4 . However, the obtained accuracy was equivalent and the method was more sensitive to noise and density fluctuations. This comparison was detailed in another Ref. 11.

The four boundary conditions are written in full in Eqs. (8)-(11). For simplicity, the positions are written as $x$, with $x_{n}$ being the reflection point to be determined, $\Delta x=x_{n}-x_{n-1}$, and the ordinate values of $f_{n} N(f, R) / c$ are written as $y_{n}$,

$$
\begin{aligned}
\Delta \phi_{x_{n-1}}^{x_{n}} & =a \frac{(\Delta x)^{3}}{3}+b \frac{(\Delta x)^{2}}{2}+c \Delta x, \\
0 & =a x_{n}^{2}+b x_{n}+c, \\
y_{n-1} & =a x_{n-1}^{2}+b x_{n-1}+c, \\
y_{n-2} & =a x_{n-2}^{2}+b x_{n-2}+c .
\end{aligned}
$$

The parameter $c$ can be isolated in Eq. (9) and inserted in Eqs. (10) and (11). Then, $b$ can be isolated in Eq. (11) and inserted in Eq. (10) to have an expression depending only on $a$. Solving this way for $a, b$, and $c$ leads to the solutions in the following equation:

$$
\begin{aligned}
& a=\frac{y_{n-1}-y_{n-2}\left(x_{n-1}-x_{n}\right) /\left(x_{n-2}-x_{n}\right)}{x_{n-1}^{2}-x_{n}^{2}-\left(x_{n-2}^{2}-x_{n}^{2}\right)\left(x_{n-1}-x_{n}\right) /\left(x_{n-2}-x_{n}\right)}, \\
& b=\frac{y_{n-2}-a\left(x_{n-2}^{2}-x_{n}^{2}\right)}{x_{n-2}-x_{n}}, \\
& c=-a x_{n}^{2}-b x_{n} .
\end{aligned}
$$

Adding the phase shift from Eq. (8) leads to

$$
a \frac{(\Delta x)^{3}}{3}+b \frac{(\Delta x)^{2}}{2}+c \Delta x-\Delta \phi_{x_{n-1}}^{x_{n}}=0
$$

with the parabola parameters $a, b$, and $c$ given by Eq. (12). This way, numerically finding the appropriate root of this complicated equation solves for $x_{n}$. Readily available tools can be implemented for this task, such as the Matlab functions fzero and fsolve.

In order to solve for all $x_{n}$, two previous positions are necessary. Therefore, these implementations start with the standard linear Bottollier-Curtet method for the first steps. The third position is also not suitable for the parabolic method. The flat derivative at the vacuum boundary enforced a strong condition for the parabola that is hard to satisfy, making the method diverge in some occasions. As such, the parabolic method is implemented starting at the fourth frequency step.

Additional constraints are also necessary to make the method stable when there are spikes in the phase shift measurement. The method is restrained to only return positions between the previous position and the linear solution (i.e., the parameter $a$ cannot be positive). If this condition is broken in any reconstruction step, the linear solution is implemented on that step.

\section{B. Square root integration shapes}

By observing an example of refractive index profile, it can be noted that the slope of the profile tends to be steeper near the reflection position, resembling a square root function. Due to this fact, a square root profile is also taken into account in this section.

If a square root function is integrated and the result is compared to a multiplication of horizontal and vertical displacements, $d x$ and $d y$, one finds that the area of a square root is equal to (2/3) $d x d y$. Therefore, assuming a square root shape in the last integration step is equivalent to using the weight factor $W$ equals to $2 / 3$, which turned out to be equivalent to the other assumptions already mentioned. ${ }^{16,17}$ The refractive index shape in this method is therefore constant across all frequency steps.

\section{C. $x^{\alpha}$ integration shapes}

In the conditions of high density, density gradient, and a small radial step, the square root function fits the refractive index almost perfectly. If these conditions are not well satisfied, which is typical of plasma edge conditions, the refractive index shape changes away from a square root function towards a step function, as depicted in Fig. 2. In order to adapt the integration shapes to these changes, this section proposes the implementation of functions of the type $x^{\alpha}$, with $\alpha$ as a function of the plasma radius. In the same manner that the integration of

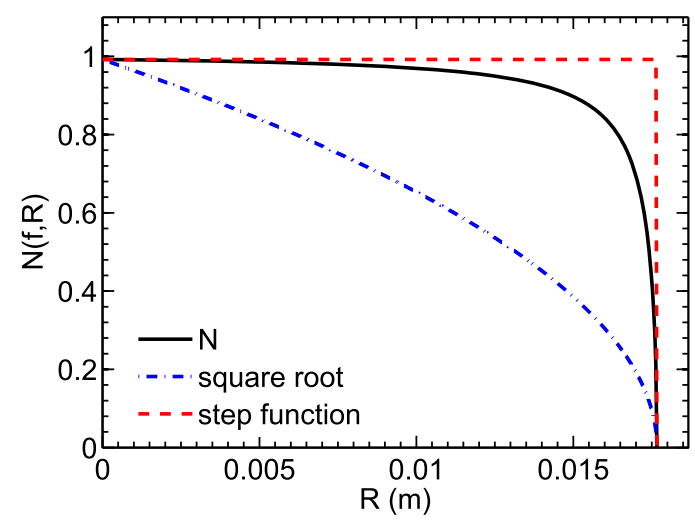

FIG. 2. The shape of the refractive index $N$ in typical edge conditions is compared to a square root and a step function. Probed in the right-hand polarization with a frequency slightly above the initialization at $f=f_{c}$. The position $\mathrm{R}=0$ marks the initialization on the plasma edge and increases towards the plasma core. 


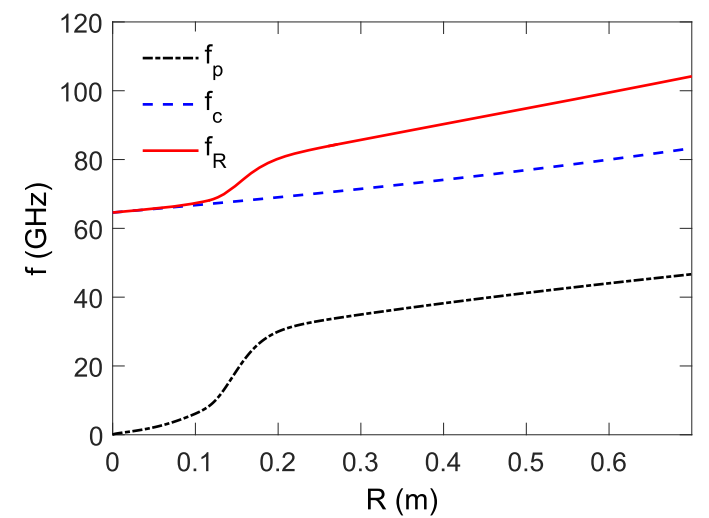

FIG. 3. Frequency profiles according to Eqs. (1) and (2) from a typical Tore Supra discharge including a pedestal located at $R=12 \mathrm{~cm}$ and $\mathrm{B}=2 \mathrm{~T}$ at the plasma center.

the square root function was shown to result in $W=2 / 3$, functions of the type $x^{\alpha}$ can be demonstrated to lead to a generic form of $W=1 /(\alpha+1)$. Therefore, the factor $W$ varies between one (when $\alpha=0$ ) and $2 / 3$ (when $\alpha=1 / 2$ ).

The accuracy of all methods listed so far is compared in Sec. III D for the cases of a linear profile in $f_{R}\left(d f_{R} / d R\right.$ is constant) and a typical scenario in Tore Supra. In this section, instead of pursuing how to find the profile of the best $x^{\alpha}$ shapes, the optimized profile of $W$ is computed for the typical Tore Supra case of Sec. III D. This way, the obtained accuracy and stability when using the optimized profile of $W$ can be compared with the other methods presented. Afterwards, in Section VI, the procedure to find the optimized $W$ profile for any profile shape is elaborated.

The Tore Supra test case of Sec. III D has a low edge density and density gradient and a pedestal at the position of $12 \mathrm{~cm}$. The frequency profiles for this example are shown in Fig. 3, assuming the low background magnetic field of $2 \mathrm{~T}$ in the plasma center. For this case, the $x^{\alpha}$ shapes, and therefore the $W$ profile, are computed following the WKB approximation and depicted in Fig. 4. Although these figures describe the behaviour of the right-hand polarization, the lefthand polarization has almost identical results. However, due to technological constraints, the left-hand polarization cannot be probed on very low densities because $f_{L}$ goes to zero. Therefore, the initial positions where the factor $W$ is farther from

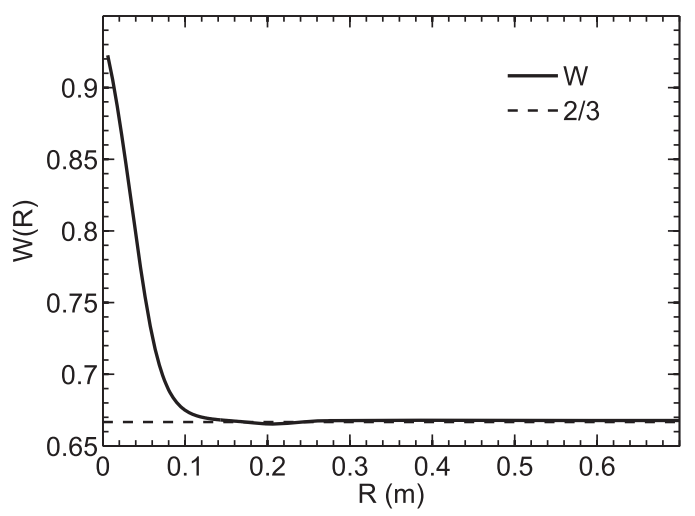

FIG. 4. Profile of weight factor W, as defined in Eq. (6), for the typical Tore Supra profile example as depicted in Fig. 3.
$2 / 3$ are not expected to be probed and the correction in $W$ is less crucial.

\section{Comparison of accuracy from all methods}

In order to analyse the performance of each method, density and magnetic field profiles are assumed and the reconstruction is simulated. The plasma size, the maximum density, and the magnetic field profile correspond to typical Tore Supra discharges. Also following the present data-acquisition characteristic, 500 probing frequencies are considered. To analyse the obtained accuracy of each method, the figures show the profile of discrepancy from the positions calculated to the correct cutoff positions for all probing frequencies. Since the magnetic field is assumed to be well known, the errors in density come from these errors in the calculation of the cutoff positions.

Initially, the simplest density profile shape is assumed: a density profile that results in a linear $f_{R}$ profile. This way, the basic characteristics of each method are visible without additional features from the profile shape.

The comparison between the obtained discrepancy profiles for the methods of linear Bottollier-Curtet, parabolic, and the square root is presented in Fig. 5. The linear BottollierCurtet solution and the parabolic methods start with the same accuracy of $\sim 1 \mathrm{~mm}$ in the first reconstruction positions. This is expected since the parabolic method is implemented starting at the fourth position. When going to the core positions, the parabolic method becomes more accurate, while the linear Bottollier-Curtet method becomes less accurate. The parabolic method showed an improvement compared to the linear Bottollier-Curtet method in the core region without needing the stabilization averaging trick introduced in the linear method. When the weight factor $W$ is taken as $2 / 3$, e.g., when the integrating shape is assumed to be a square root, the error in the edge region is just above half millimeter and quickly converges close to $0.3 \mathrm{~mm}$ across the entire profile.

Having in mind these main characteristics, the reconstruction of a more complex density profile shape can be simulated to emphasize some features and demonstrate other features. The next example is a density profile shape routinely observed

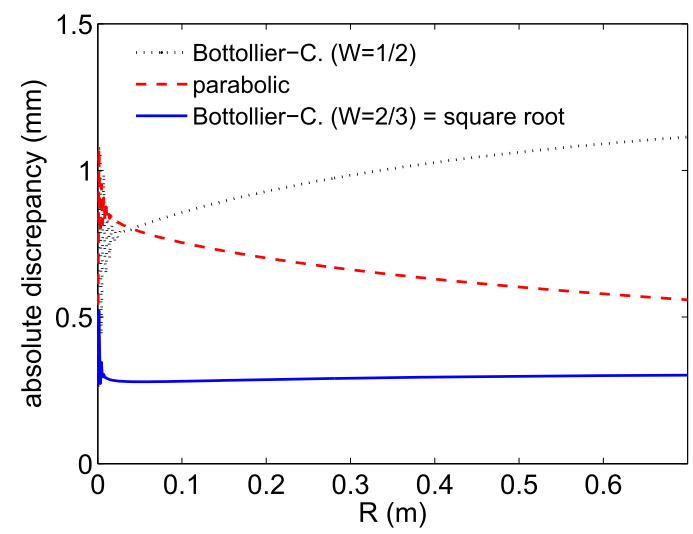

FIG. 5. Profiles of absolute radial discrepancy when reconstructing the density profile with the conditions of linear $f_{R}$ profile, 500 probing frequencies, and a precision of $0.1 \mathrm{~mm}$ in the initialization. 


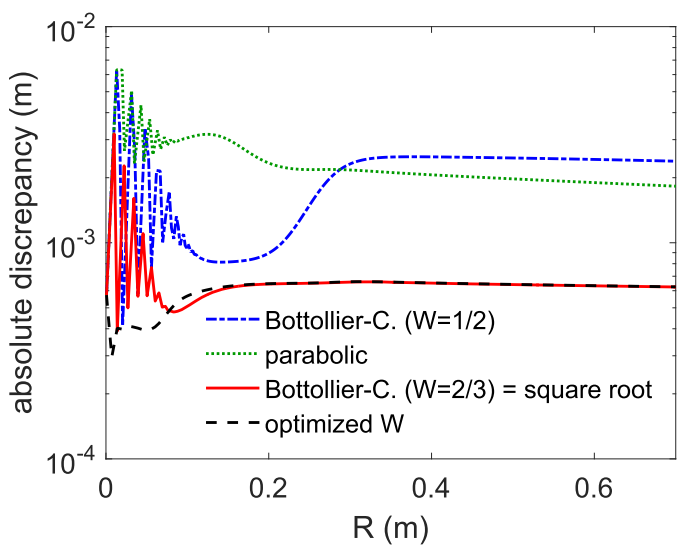

FIG. 6. Profiles of absolute radial discrepancy when reconstructing the density profile with the conditions of 500 probing frequencies, a precision of 0.1 $\mathrm{mm}$ in the initialization, and the Tore Supra example profiles as depicted in Fig. 3.

in Tore Supra and other latest generation tokamaks. It consists of a low density and density gradient in the edge region, followed by a pedestal with high density gradient and back to low density gradient around the plasma center. In this case, the method with the integration shapes $x^{\alpha}$ optimized for this profile shape is also included in the comparison, which can be found in Fig. 6.

In Fig. 6, the parabolic method does not show an improvement from the linear Bottollier-Curtet method. Reinforcing the feature observed before, the parabolic shape is especially inaccurate to describe the refractive index shape at edge conditions.

The method with the square root shape shows more features in this case. First, the edge region, where the plasma has a low density and density gradient, is less accurate, with oscillations peaking up to $3.2 \mathrm{~mm}$. Then, the valley around $12 \mathrm{~cm}$ marks the pedestal, where the density gradient is at maximum. When the density gradient increases, the radial step decreases for a fixed frequency step, resulting in a more accurate determination of the probing positions. In addition, the refractive index profile is better described by a square root shape at the core region, due to higher density and density gradient, and when smaller radial steps occur, i.e., the approximation used in Bottollier-Curtet Ph.D. thesis. ${ }^{16}$

If the refractive index shape is adapted for the edge region with the shape $x^{\alpha}$ as introduced in Section III C, the edge inaccuracy and oscillations from the square root method disappear. This method achieved a precision level around $0.6 \mathrm{~mm}$ through the entire profile. In practice, the reconstruction accuracy in this case will be determined by the noise and turbulence levels and the precision in the initialization. The method to estimate this implemented profile of the integration weight factors $W$ is elaborated in Section VI.

\section{STABILITY OF THE INVERSION METHOD}

The linear Bottollier-Curtet's algorithm (when $W=1 / 2$ ) turned out to be unstable and a stabilization mechanism was introduced. The measured phase shift of each frequency step is averaged with the value of the previous step. In order to analyse the stabilization mechanisms, the expression used to calculate each new position from Eq. (6) is rewritten in the following equation, for a simpler referencing,

$$
\Delta x=\frac{\Delta \phi}{W N}
$$

with $\Delta x$ being the radial step normalized by the probing wavelength $\left(x_{n}-x_{n-1}\right) / \lambda_{n}, \Delta \phi$ as given by Eq. (7), normalized by $2 \pi$, and $N$ being $N\left(f_{n}, x_{n-1}\right)$.

First, the averaging with the previous step, as in the linear Bottollier-Curtet solution, was tested for every component of Eq. (14) (the radial step, the phase shift, and the value of $N$ ). The conclusion is that there is no preferred component to be averaged. The best component to average varies depending on the profile to be reconstructed. Next, the numerical stability is investigated with a fixed local integration factor $W$ which is always the case apart from the parabolic method, where a new parabola is computed in each step.

To investigate the role of each element of Eq. (14) in the stability of the reconstruction method, the evolution of the error in each of these elements was observed along a reconstruction. This is possible since the correct positions and profiles are known. First, it was noted that the error in the evaluation of $N\left(x_{n-1}\right)$ and $\Delta \phi_{x_{n-1}}^{x_{n}}$ comes from an error in the determination of $x_{n-1}$, meaning that an error in the position $x_{n}$ is due to not using the ideal value for $W$, plus the error propagated from the previous position into $\Delta \phi$ and $N$. The propagation of these error sources in $\phi, W$, and $N$ can be evaluated by equating them as in Eqs. (15)-(17), respectively. The error in $\Delta \phi$ is additive, whereas in $W$ and $N$, the error is introduced as a multiplicative factor $\epsilon$ and $\zeta$, respectively,

$$
\begin{aligned}
& \frac{1}{W} \frac{\left(\Delta \phi+\Delta \phi^{\prime}\right)}{N}=\frac{1}{W} \frac{\Delta \phi}{N}+\frac{1}{W} \frac{\Delta \phi^{\prime}}{N}, \\
& \frac{1}{\epsilon W} \frac{\Delta \phi}{N}=\frac{1}{W} \frac{\Delta \phi}{N}+\left(\frac{1-\epsilon}{\epsilon}\right) \frac{1}{W} \frac{\Delta \phi}{N}, \\
& \frac{1}{W} \frac{\Delta \phi}{\zeta N}=\frac{1}{W} \frac{\Delta \phi}{N}+\frac{1}{W}\left(\frac{1-\zeta}{\zeta}\right) \frac{\Delta \phi}{N} .
\end{aligned}
$$

Equating these error sources shows how each element influences the error propagation. All steps are subject to the error of not using the ideal local value of $W$, plus the error propagating. The errors in the evaluation of the phase shift will be multiplied by the factor $1 / W N$, whereas the errors in the refractive index and weight factor are multiplied by $\Delta \phi / W N$. Furthermore, any error in each element of Eq. (14) induces an oscillation. For example, an excess in $x_{n}$ causes an excess in the computed $\Delta \phi_{0}^{n}$ for the frequency $f_{n+1}$, which causes an under evaluation of $\Delta \phi_{n}^{n+1}$, resulting in under evaluating $x_{n+1}$. These errors are damped or not depending on the error multiplicative factors written in Eqs. (15)-(17).

The combination of these facts shows that the more efficient scenario to damp errors is when $W N$ is greater because the error is multiplied by $1 / W N$ in the next step. Furthermore, the reconstruction damps the previous errors when $1 / W$ is between one and two (the step function and linear cases). For $1 / W$ equals or greater than two, there is no damping of errors. These conclusions come from the oscillatory character explained. An 
error of $+E$ in one step translates into an error of $-E$ in the next step. When this error is multiplied by one, the best damping occurs because $+E-1 \times E=0$. When it is multiplied by two, no damping occurs because $+E-2 \times E=-E$. Since there is no error damping and an additional error is introduced in every step by not using the perfect value of $W$, the system becomes unstable. This interpretation explains why it is observed that the square root integration shape converges, unlike the linear integration, and when the factor $W$ and $N$ are closer to one, any error introduced is damped more efficiently. This last feature is observed in the edge conditions in Sec. V. A last remark is that this analysis is done focusing on the true local values of $W$. Using $W=1$ when it is not the ideal value can be interpreted as if the damping efficiency is not of $W=1$ but of the ideal local value itself. In addition, an error is introduced from the excess in $W$, as it was shown in Eq. (16).

\section{NOISE ANALYSIS}

This section demonstrates how different sources of noise impact the reconstruction accuracy. First, a spike of $1 \%$ is added in the phase shift of a single frequency. The radial error at the location where the phase shift spike was introduced is presented in Fig. 7.

Fig. 7 shows that an error introduced is damped faster for the square root method. The parabolic method enforced boundary conditions to find the parabolas and this made the error propagate longer. The fluctuations stay even longer if the parabolas are computed using the boundary condition of a constant derivative at the previous point. A spike in the probing frequency and a density perturbation were also tested. However, the comparison between the different methods had the same features as presented in the phase shift case in Fig. 7.

Next, white noise is added to the phase shift data, as a percentage of the average value, to simulate the experimental noise in the Tore Supra example. Fig. 8 shows the obtained accuracies for the reconstruction methods with fixed $W=2 / 3$ and optimized $W$ profile, at two different noise levels. From the interpretations in Sec. IV, one can observe how the edge region is very efficient in damping the noise introduced since $1 / W N$ is

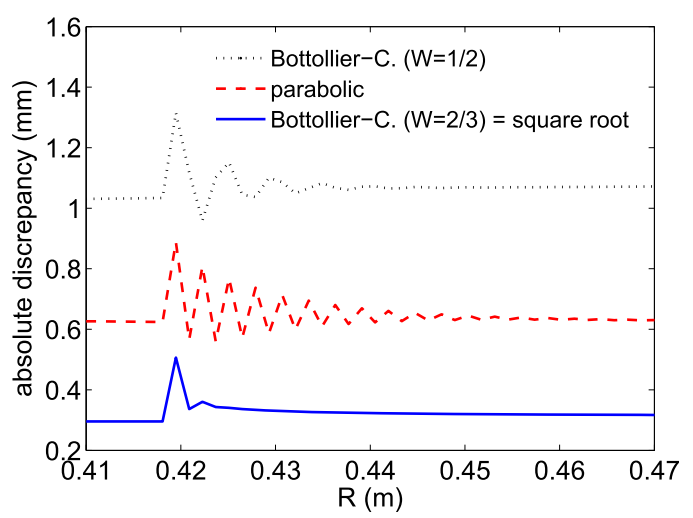

FIG. 7. Radial discrepancy when reconstructing the density profile with a $1 \%$ spike in the phase shift of a single frequency and conditions of 500 probing frequencies, a precision of $0.1 \mathrm{~mm}$ in the initialization, and the linear $f_{R}$ profile.

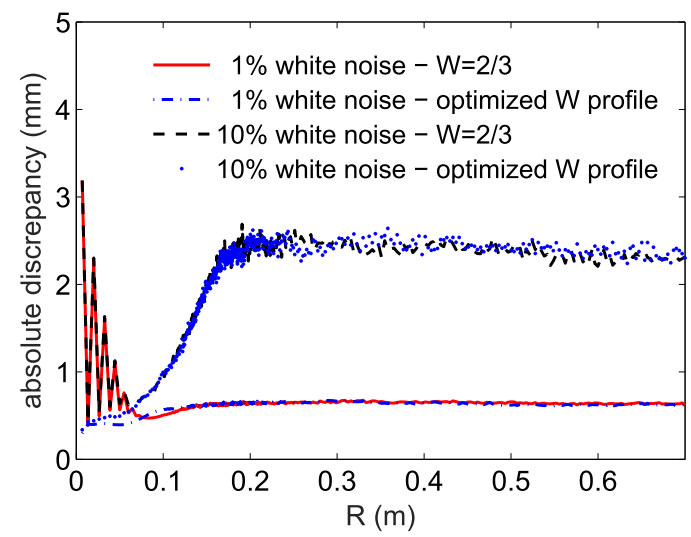

FIG. 8. Radial discrepancy when reconstructing the density profile with different levels of white noise in the phase shift data and conditions of 500 probing frequencies, a precision of $0.1 \mathrm{~mm}$ in the initialization and, the typical low B Tore Supra example profile. Each discrepancy profile is the average of 500 reconstructions.

minimized. This is an interesting feature of the reconstruction method since the edge region is typically more prone to noise and fluctuations in tokamaks. In the core region, on the other hand, the discrepancy saturates at a higher value, depending on the noise level. All methods showed the exact same tendency. They retain the same discrepancies in the edge region for all noise levels and linearly increase in the core as the noise level increases. The parabolic method is not represented because it was able to find the parabolas only with a maximum noise level up to $1 \%$. Beyond this level of noise, the method is unable to associate parabolas for the data in too many cases and the method collapses to the linear solution.

\section{FINDING THE IDEAL REFRACTIVE INDEX SHAPE FROM THE PLASMA PROPERTIES}

The analyses in Secs. III-V demonstrate the accuracy and stability improvements when optimizing $W$ everywhere. In this section, the procedure to find the profile of the optimized $W$ is elaborated.

For the simplified Tore Supra example used in Secs. III-V, the profile of $W$ was given in Fig. 4. In this case, one can implement a linear fit in $W$, from the edge value to $2 / 3$ in the pedestal position. The precision of the pedestal position was tested to not be crucial for the obtained accuracy. The bottlenecks of this implementation are the edge $W$ value and the validity of the assumed shape, i.e., a well defined transition from low density and gradient to high gradient. The initial $W$ value can start at one and be improved either iteratively or by the expression in Eq. (28) developed below. Nevertheless, an initial reconstruction is necessary to estimate an initial profile of $\nabla f_{R}$ for computing the expression in Eq. (28) and this method provides a good initial reconstruction of the density profile.

The next step is to determine the profile of refractive index shape, which gives $W$, based on the local plasma properties. The first and most natural step is to observe what is the best function of the type $x^{\alpha}$ to fit the refractive index profile at different probing positions depending on the integration length. For the example Tore Supra profile, these relations are presented in Fig. 9. 


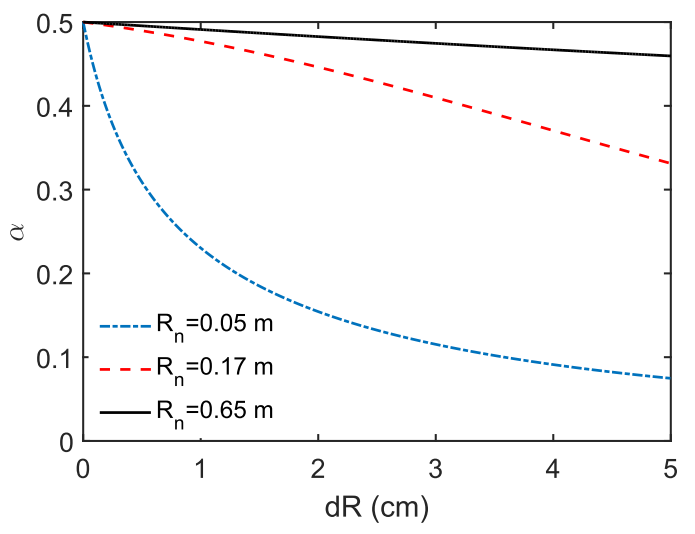

FIG. 9. Optimized exponent of the refractive index shape function, as $x^{\alpha}$ by the radial integration length at three example radial positions for the example of typical Tore Supra profiles given by Fig. 3 .

At all plasma positions, the value of $\alpha$ converges to $1 / 2$ for $d R$ going to zero. As the reconstruction step $d R$ increases, $\alpha$ goes to zero depending on the local plasma parameters. The first approach would be to fit the evolution of $\alpha$ versus $d R$ and relate the fitting parameters to the local plasma properties. Nevertheless, two major difficulties arise when doing so. First, the step length is not yet known in a reconstruction step. It is actually the quantity to be calculated. Second, the fitting parameters do not have a straightforward relation to fit against the plasma properties. To solve these problems, the relations of $\alpha$ can be expressed in terms of a known quantity. For example, the step in the probing frequency or the refractive index value at the previous position $x_{n-1}$, i.e., $N_{n-1}$. The refractive index was chosen over the frequency step because the relations are simpler to fit. Since the refractive index is zero at the reflection position and goes towards one as the reconstruction step increases, the parameter $\alpha$ can be adjusted taking this range into account, as in the proposed shape function in Eq. (18):

$$
x^{\alpha}=x^{0.5-0.5 \beta\left(N_{n-1}\right)} .
$$

In order to solve for $\beta$, it is assumed that the plasma parameters that dictate the refractive index shape are the local cutoff frequency, $f_{R}$, (i.e., the probing frequency) and the gradient of the frequency cutoff profile, $\nabla f_{R}$.

In Fig. 10, three example relations for three different probing positions in the same example Tore Supra conditions for a $5 \mathrm{~cm}$ radial step are shown. When probing the plasma core, since the density in the previous probing position is not close to vacuum, the refractive index $N_{n-1}$ is small, which makes the relation $\beta\left(N_{n-1}\right)$ well described by a linear function of $N_{n-1}^{2}$. This is seen in Fig. 10 for the position of $0.65 \mathrm{~m}$. On the other hand, when probing in the edge plasma with low density, as also exemplified in Fig. 10, the refractive index is close to one and the $\beta\left(N_{n-1}\right)$ behaviour in this region needs a correction from the linear solution. In addition, the same pair $\left(f_{R}, \nabla f_{R}\right)$ from the position $0.65 \mathrm{~m}$ of the example case in Fig. 10 could appear in the edge region of a different profile.

Therefore, to ensure that the solution obtained is valid for any profile in general, the $\beta$ relations are mapped over a wide range of $f_{R}$ and $\nabla f_{R}$, while still ensuring that the entire range of refractive index is taken into account. To achieve this,

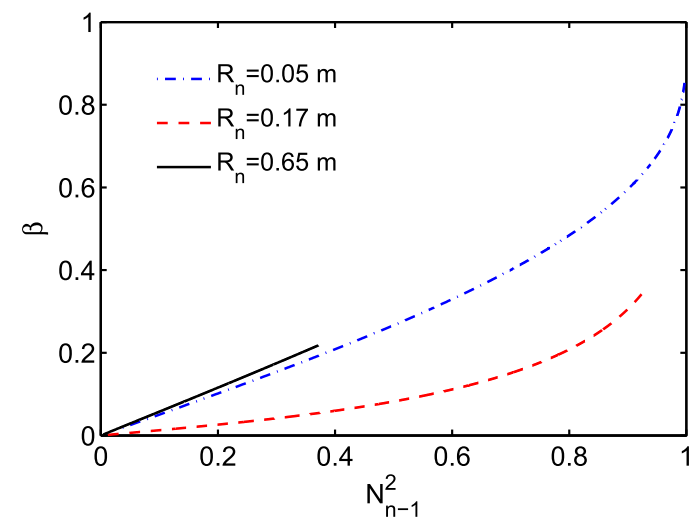

FIG. 10. The $\beta$ parameter from the refractive index fitting shape given in Eq. (19), as a function of $N_{n-1}^{2}$, taken at three example radial positions for the example of typical Tore Supra profiles as given in Fig. 3.

new density and magnetic field profiles are assumed for each value of $f_{R}, \nabla f_{R}$. Furthermore, the fittings are separated into two refractive index ranges. First, the linear regime is fitted with $N_{n-1}$ from zero to 0.25 , and after, the correction on the linear solution with $N_{n-1}$ from zero to one. The distinction is necessary because the boundary conditions of each regime are different and are explained in detail bellow. The dependency on $\nabla^{2} f_{R}$ is neglected. Thus, the assumed profiles are enforced with a radially constant $\nabla f_{R}$. The $\nabla f_{R}$ is a combination of $\nabla f_{p e}$ and $\nabla f_{c e}$, but $\nabla f_{c e}$ has a positive curvature fixed by the background magnetic field. This results in a negative $\nabla f_{p e}$ for a constant $\nabla f_{R}$. If $\nabla f_{R}$ is too low, enforcing a constant $\nabla f_{R}$ profile results in negative values for the electron density, which is unphysical. When solving the linear regime, the initial density can be increased as necessary and the density profile can have a negative gradient while having positive density and constant $\nabla f_{R}$. The minimum value of $\nabla f_{R}$ achieved when fitting the linear regime was $8 \mathrm{GHz} / \mathrm{m}$. In any case, if $\nabla f_{R}$ goes to zero, the WKB solution of a sharp reflection also breaks down and full wave effects would have to be considered. The upper limit solved was $200 \mathrm{GHz} / \mathrm{m}$, but it is not important since at very high gradients, the step size is negligible, $N_{n-1}$ goes to zero, and the square root solution is very precise. The solved probing frequency range, $f_{R}$, is from $35 \mathrm{GHz}$ to $250 \mathrm{GHz}$. Below this value, the fitting shapes assumed start to be less adequate, and above it, it is not possible to ensure the constant $\nabla f_{R}$.

After the linear regime is solved, the correction part is fitted using a different sweeping tactic. This is because the initial density cannot be increased to compensate for the negative density profile curvature. All density profiles assumed start with vacuum in the edge to ensure the range of $N_{n-1}$ until the value of one. The minimum $\nabla f_{R}$ solved in this case was frequency dependent and increases linearly from $12 \mathrm{GHz} / \mathrm{m}$, when probing at $35 \mathrm{GHz}$, up to $40 \mathrm{GHz} / \mathrm{m}$, when probing at $120 \mathrm{GHz}$. Even though the ranges are shorter for the correction part, it is still a broad range because the correction is only relevant near the initialization frequencies, when $N_{n-1}$ is much closer to one. The corrections were fitted assuming the shape $a N_{n-1}^{b}$. The value of $b$ does not vary much and therefore it was fixed at 3.44 , or 4.44 , if related to $N_{n-1}$ not squared. Therefore, the relations of $\beta\left(N_{n-1}\right)$ can now be written in full 


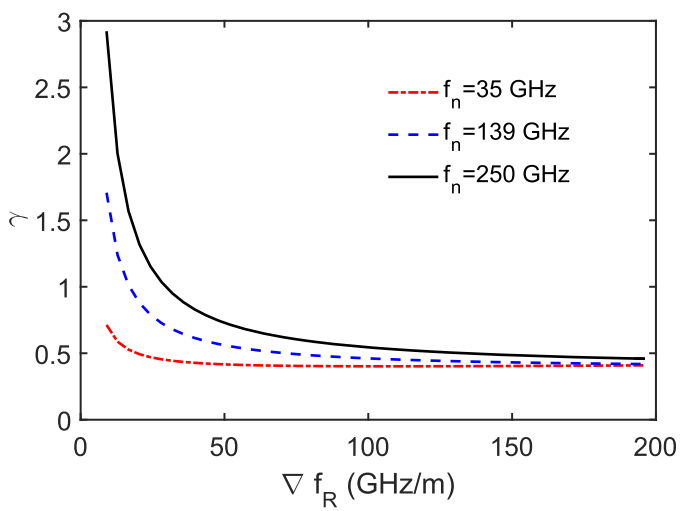

FIG. 11. The $\gamma$ parameter from the refractive index fitting shape, as given by Eq. (19), versus the cutoff profile gradient.

as

$$
x^{\alpha}=x^{0.5-0.5 \beta\left(N_{n-1}\right)}=x^{0.5-0.5\left(\gamma N_{n-1}^{2}+\delta N_{n-1}^{4.44}\right)} .
$$

These relations of $\gamma$ and $\delta$ can be found in Figs. 11 and 12 , respectively. The linear relations are fitted with functions of the following expression:

$$
\gamma\left(f_{R}, \nabla f_{R}\right)=p_{1}\left(f_{R}\right) /\left(\nabla f_{R}-p_{2}\left(f_{R}\right)\right)+p_{3}\left(f_{R}\right) .
$$

The correction part is fitted with the same shape, but with parameters named with $q$, as

$$
\delta\left(f_{R}, \nabla f_{R}\right)=q_{1}\left(f_{R}\right) /\left(\nabla f_{R}-q_{2}\left(f_{R}\right)\right)+q_{3}\left(f_{R}\right) .
$$

Using this function shape, all fitted relations have an accuracy around $1 \%$ for all probing frequencies. The last step is to know the parameters $p_{n}$ and $q_{n}$ as a function of $f_{R}$. They are smooth well-behaved functions of $f_{R}$ and well fitted by high order polynomials, as given by Eqs. (22)-(27). The quality of the fit is expressed as the RMS value of the residuals, computed as a percentage to the average value of that parameter,

$$
\begin{aligned}
p_{1}= & a_{1} f_{R}^{2}+b_{1} f_{R}+c_{1}, \\
p_{2}= & a_{2} f_{R}^{6}+b_{2} f_{R}^{5}+c_{2} f_{R}^{4}+d_{2} f_{R}^{3}+e_{2} f_{R}^{2} \\
& +f_{2} f_{R}+g_{2}, \\
p_{3}= & a_{3} f_{R}^{4}+b_{3} f_{R}^{3}+c_{3} f_{R}^{2}+d_{3} f_{R}+e_{3},
\end{aligned}
$$

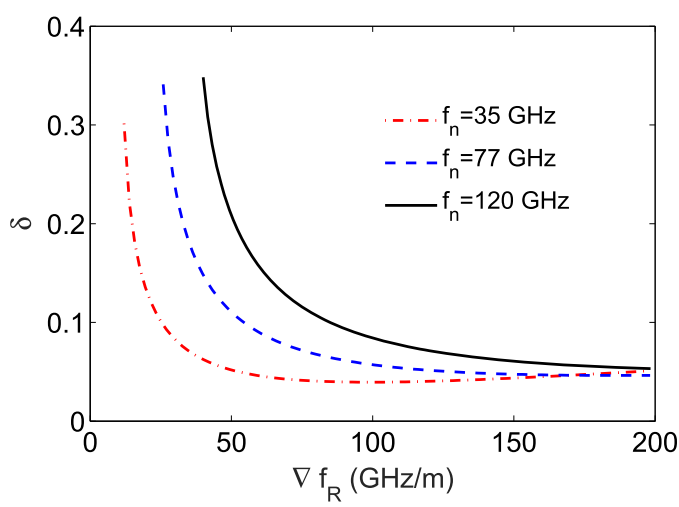

FIG. 12. The $\delta$ parameter from the refractive index fitting shape, as given by Eq. (19), versus the cutoff profile gradient.
TABLE I. Fitted $p_{1}, p_{2}$, and $p_{3}$ parameters with $f_{R}$ in GHz.

\begin{tabular}{lll}
\hline \hline \multicolumn{1}{c}{$\mathrm{p}_{1}$} & \multicolumn{1}{c}{$\mathrm{p}_{2}$} & \multicolumn{1}{c}{$\mathrm{p}_{3}$} \\
\hline RMS $=0.3 \%$ & $\mathrm{RMS}=0.5 \%$ & $\mathrm{RMS}=0.04 \%$ \\
$\mathrm{a}_{1}=-3.251 \times 10^{-5}$ & $\mathrm{a}_{2}=6.423 \times 10^{-13}$ & $\mathrm{a}_{3}=3.098 \times 10^{-11}$ \\
$\mathrm{~b}_{1}=0.08323$ & $\mathrm{~b}_{2}=-6.247 \times 10^{10}$ & $\mathrm{~b}_{3}=-2.148 \times 10^{-8}$ \\
$\mathrm{c}_{1}=-1.136$ & $\mathrm{c}_{2}=2.476 \times 10^{-7}$ & $\mathrm{c}_{3}=5.937 \times 10^{-6}$ \\
& $\mathrm{~d}_{2}=-5.136 \times 10^{-5}$ & $\mathrm{~d}_{3}=-7.614 \times 10^{-4}$ \\
& $\mathrm{e}_{2}=5.940 \times 10^{-3}$ & $\mathrm{e}_{3}=0.3843$ \\
& $\mathrm{f}_{2}=-0.3676$ & \\
& $\mathrm{~g}_{2}=11.43$ & \\
\hline \hline
\end{tabular}

TABLE II. Fitted $q_{1}, q_{2}$, and $q_{3}$ parameters with $f_{R}$ in GHz.

\begin{tabular}{lll}
\hline \hline \multicolumn{1}{c}{$\mathrm{q}_{1}$} & \multicolumn{1}{c}{$\mathrm{q}_{2}$} & \multicolumn{1}{c}{$\mathrm{q}_{3}$} \\
\hline RMS $=0.02 \%$ & $\mathrm{RMS}=0.1 \%$ & $\mathrm{RMS}=0.04 \%$ \\
$\mathrm{a}_{1}^{\prime}=2.237 \times 10^{-7}$ & $\mathrm{a}_{2}^{\prime}=-6.599 \times 10^{-5}$ & $\mathrm{a}_{3}^{\prime}=-8.132 \times 10^{-12}$ \\
$\mathrm{~b}_{1}^{\prime}=-6.005 \times 10^{-5}$ & $\mathrm{~b}_{2}^{\prime}=0.2252$ & $\mathrm{~b}_{3}^{\prime}=3.746 \times 10^{-9}$ \\
$\mathrm{c}_{1}^{\prime}=0.0430$ & $\mathrm{c}_{2}^{\prime}=0.3522$ & $\mathrm{c}_{3}^{\prime}=-6.902 \times 10^{-7}$ \\
$\mathrm{~d}_{1}^{\prime}=-0.3808$ & & $\mathrm{~d}_{3}^{\prime}=6.360 \times 10^{-5}$ \\
& & $\mathrm{e}_{3}^{\prime}=-2.948 \times 10^{-3}$ \\
& & $\mathrm{f}_{3}^{\prime}=0.08253$ \\
\hline
\end{tabular}

$$
\begin{aligned}
& q_{1}=a_{1}^{\prime} f_{R}^{3}+b_{1}^{\prime} f_{R}^{2}+c_{1}^{\prime} f_{R}+d_{1}^{\prime}, \\
& q_{2}=a_{2}^{\prime} f_{R}^{2}+b_{2}^{\prime} f_{R}+c_{2}^{\prime}, \\
& q_{3}=a_{3}^{\prime} f_{R}^{5}+b_{3}^{\prime} f_{R}^{4}+c_{3}^{\prime} f_{R}^{3}+d_{3}^{\prime} f_{R}^{2}+e_{3}^{\prime} f_{R}+f_{3}^{\prime}
\end{aligned}
$$

with all parameters $a_{i}$ to $g_{i}$ given in Table I and $a_{i}^{\prime}$ to $f_{i}^{\prime}$ in Table II.

Last, the optimized integration weight factors $W$ can be written, in full, as

$$
W\left(N_{n-1}, f_{R}, \nabla f_{R}\right)=\frac{1}{\alpha\left(N_{n-1}, f_{R}, \nabla f_{R}\right)+1},
$$

with $\alpha\left(N_{n-1}, f_{R}, \nabla f_{R}\right)$ given by Eq. (19), and $\gamma$ and $\delta$ given by Eqs. (20-27) and Tables I and II.

This final expression allows optimizing the weight factors $W$ at all frequency steps and achieving the accuracy shown in Section III D, Fig. 6, for any profile shape that satisfies the

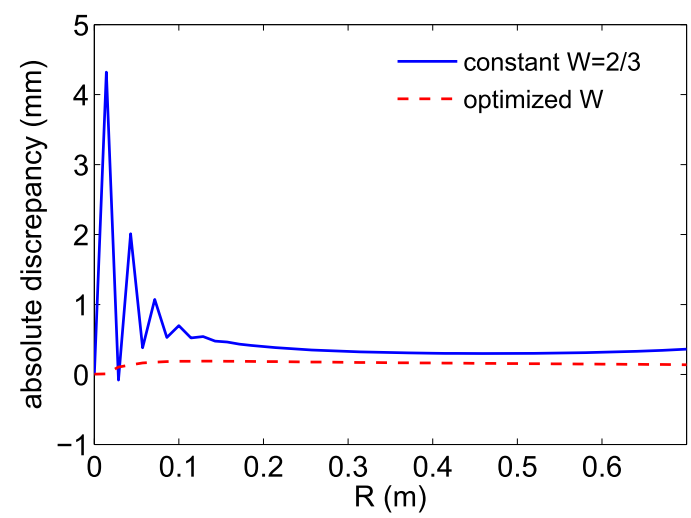

FIG. 13. Example of the optimization of the weight factor $W(R)$, as defined in Eq. (6), for a linear $f_{R}$ profile and only 50 probing frequencies. 
boundary conditions explained in this section. In Sec. VII, Eq. (28) and the parameters in Tables I and II are used to optimize the $W$ profile and reconstruct the density profile of a worst case example, given in Fig. 13, including the complication of using less frequency steps.

\section{RECONSTRUCTIONS WITH LESS PROBING FREQUENCIES}

Up to now, all profile reconstructions obeyed the rule of more probing frequencies resulting in better accuracy, which is mainly because the refractive index shape is better described by a square root function the smaller is the radial step. In addition, the error introduced by miscalculating the area under the refractive index curve is proportionally bigger to the increase of the radial step, emphasizing the previous feature. With the results of Sec. VI, however, the mismatch in the shape of the refractive index can be corrected. Therefore, the profile can be reconstructed using less probing frequencies without a significant loss of accuracy.

Using less probing frequencies speeds up the reconstruction algorithm, allowing for monitoring of faster density profile evolution. This was tested for the Tore Supra example profile when reducing the number of frequencies from 500 to 100 and speeding the reconstruction for roughly about five times, depending on the computer conditions. In this case, the fluctuations in the edge region peak at $1 \mathrm{~cm}$ when the factor $W$ is fixed at $2 / 3$. When $W$ is optimized for all steps, the discrepancy profile goes back to values below $1 \mathrm{~mm}$.

Other situations that the reconstruction accuracy is significantly improved by optimizing the $W$ factor are scenarios with very low or very high background magnetic fields. The refractive index in each previous positions, $N_{n-1}$, is closer to one the greater is the difference between $f_{c e}$ and $f_{p e}$, which causes the factor $W$ to increase resulting in a degradation of the reconstruction accuracy when using a fixed $W$, as in the square root method. To demonstrate one case, a low density profile linear in $f_{R}$ with $\nabla f_{R}=20 \mathrm{GHz} / \mathrm{m}$ is assumed. In this condition, no pedestal is present and it is not clear where the square root function describes well the refractive index shape. In addition, the number of probing frequencies is reduced to 50. Applying the method developed in Sec. VI gives the optimized $W$ for each probing frequency. In this case, the $W$ profile is never too close to the square root method solution of $2 / 3$. The $W$ profile is always above 0.7 . The accuracy of the reconstructed profiles before and after optimizing the $W$ profile is depicted in Fig. 13. A maximum error of $0.4 \mathrm{~mm}$ is observed in the reconstructed profile when the $W$ profile is optimized. If reducing even more the number of probing frequencies, the radial steps become too large. In such cases, the error in the trapezoidal integration before the last step surpasses the error from integrating the last radial step. For all cases explored here, the error in the trapezoidal integration surpasses the error in the last step when the radial step surpasses $5 \mathrm{~cm}$.

The reduction in the number of frequency steps was also tested in the Tore Supra example with the introduction of white noise in the time of flight and phase shift data. When a low level of white noise is introduced, up to $1 \%$, no difference is observed between the cases with 500 or 100 frequencies.
For higher levels of noise, the discrepancy in the core raises, doubling the noiseless value at around $10 \%$ noise level.

\section{CONCLUSIONS}

Following the recent advances and demands in the reflectometry techniques, deeper understanding and additional improvements in the density profile reconstruction method were aimed here in the data analysis front. A crucial point in the reconstruction method is the assumed refractive index shape in the integration over each radial step. It was demonstrated that using the radially optimized fractional power functions improved the reconstructed profile accuracy and stability because these functions describe well the true refractive index shape. Although the use of a square root profile achieved good results in the core plasma, it is not the best suited shape in the edge plasma. In this region, the power of the fractional power functions can be very close to zero in the first reflected frequencies. The adaptation of the power of these functions according to the plasma profile showed an accuracy improvement in the edge plasma profile of up to two orders of magnitude. The comparison between all methods also showed how the reconstruction is more stable when not using any information of the previously calculated positions to determine the next position, or even, forcing smoother variation on any of the reconstruction terms. All these procedures were demonstrated to delay the error damping capability of the reconstruction method when spurious events and phase noise were introduced. In addition, the analysis on the reconstruction stability deduced a better damping of errors for higher values of $W N_{n-1}$, which is typical of edge conditions.

When reconstructing a well known density profile shape containing a clear pedestal transition, the optimized integration shapes could be easily proposed. The bottlenecks of this implementation are: an evident transition to the pedestal, and the accuracy of the integration factor assumed in the first reconstruction step. The method elaborated in Section VI suppresses these issues. It can be used to determine the entire profile of integration factors for any profile shape. Optimizing the integration factors also allows us to use a reduced number of probing frequencies to reconstruct the profile without any accuracy loss, as demonstrated in two examples. This feature enables the real-time monitoring of faster density profile evolution.

The demonstration of all shown improvements in experimental data is beyond the scope of this paper and extended applications will be the aim of another paper.

\section{ACKNOWLEDGMENTS}

The work presented was undergone with the support of the Brazilian National Council for Scientific and Technological Development (CNPq).

\footnotetext{
${ }^{1}$ E. J. Doyle, in 12th International Reflectometry Workshop, Oral contribution, Jülich, Germany, 2015.

${ }^{2}$ C. Laviron, A. J. H. Donné, M. E. Manso, and J. Sanchez, Plasma Phys.
} Controlled Fusion 38, 905-936 (1996). 
${ }^{3}$ E. Mazzucato, Rev. Sci. Instrum. 69, 2201 (1998).

${ }^{4}$ F. Clairet, C. Bottereau, J. M. Chareau, and R. Sabot, Rev. Sci. Instrum. 74, 1481 (2003).

${ }^{5}$ F. Clairet, B. Ricaud, F. Briolle, S. Heuraux, and C. Bottereau, Rev. Sci. Instrum. 82, 083502 (2011).

${ }^{6}$ B. Baiocchi, C. Bourdelle, C. Angioni, F. Imbeaux, A. Loarte, M. Maslov, and JET Contributors, Nucl. Fusion 55, 123001 (2015).

${ }^{7}$ R. Sakamoto, B. Pégourié, F. Clairet, A. Géraud, C. Gil, S. Hacquin, and F. Köchl, Nucl. Fusion 53, 063007 (2013).

${ }^{8}$ A. J. H. Donné, J. C. van Gorkom, V. S. Udintsev, C. W. Domier, A. KrämerFlecken, N.C. Luhmann, Jr., F. C. Schüller, and TEXTOR Team, Phys. Rev. Lett. 94, 085001 (2005).

${ }^{9}$ S. J. Zweben, J. A. Boedo, O. Grulke, C. Hidalgo, B. LaBombard, R. J. Maqueda, P. Scarin, and J. L. Terry, Plasma Phys. Controlled Fusion 49, S1-S23 (2007).

${ }^{10}$ R. B. Morales, S. Hacquin, S. Heuraux, and R. Sabot, in Seminaire de l'école Doctoral EMMA, Poster contribution, Metz, France, 2015.
${ }^{11}$ R. B. Morales, S. Hacquin, S. Heuraux, and R. Sabot, in Proceedings of the 12th International Reflectometry Workshop, Jülich, Germany, 2015.

${ }^{12}$ R. B. Morales, S. Hacquin, S. Heuraux, and R. Sabot, in Proceedings of the 43rd European Physical Society Conference on Plasma Physics, Leuven, Belgium, 2016 (ECA (Europhysics Conference Abstracts), 2016), Vol. 40A, p. O4.133, available at http://ocs.ciemat.es/EPS2016PAP/html/.

${ }^{13}$ F. Chen, Introduction to Plasma Physics and Controlled Fusion (Springer, US, 1984).

${ }^{14}$ H. Bottollier-Curtet and G. Ichtchenko, Rev. Sci. Instrum. 58, 539 (1987).

${ }^{15} \mathrm{I}$. H. Hutchinson, Principles of Plasma Diagnostics (Cambridge University Press, UK, 2002).

${ }^{16} \mathrm{H}$. Bottollier-Curtet, "Réflectométrie hyperfréquence pour la détermination de la densité électronique et de ses fluctuations sur le Tokamak Petula-B," Ph.D. thesis, Université de Paris XI, 1986.

${ }^{17}$ D. A. Shelukhin, V. A. Vershkov, D. V. Sarychev, A. A. Petrov, V. G. Petrov, and G. F. Subbotin, in Proceedings of the 11th International Reflectometry Workshop, Palaiseau, France, 2011. 